\title{
Notch signaling regulates function of human mucosal-associated invariant T (MAIT) cells
}

\author{
Pimpayao Sodsai, ${ }^{1,2}$ Siriwan Klinchanhom, ${ }^{1,2}$ Nattiya Hirankarn, ${ }^{1,2}$ Rangsima Reantragoon, ${ }^{1,2}$ Tanapat Palaga ${ }^{1,3}$
}

\begin{abstract}
Background: Notch signaling plays an important role in the development of T lymphocytes and regulates their effector functions. The regulatory roles of Notch signaling on $\mathrm{T}$ cells have been intensely investigated, but whether it involves in effector functions of mucosal-associated invariant T (MAIT) cells has never been reported.
\end{abstract}

Objective: To elucidate the expression profiles of Notch receptors/ligands and to investigate their roles in human MAIT cell function.

Methods: Peripheral blood mononuclear cells (PBMCs) from healthy donors were stimulated with or without anti-CD3/CD28-coupled beads, recombinant IL-12/IL-18 cytokines, riboflavin- or non-riboflavin-synthesizing bacterial cultured supernatant for 24 hours. The expression profiles of Notch receptors and ligands on MAIT cells were detected by flow cytometry. PBMCs were treated with a Notch signaling inhibitor, gamma secretase inhibitor (GSI), before stimulation to investigate the impact of interference with Notch signaling on activation and function of MAIT cells.

Results: Resting MAIT cells predominantly expressed Notch2 receptor and the ligand, Jagged 2, on their surface. Upon stimulation, MAIT cells further upregulated Notch2 and also Notch1 with its cleaved form, indicating active Notch signaling. Cytokines and cytotoxic molecules which are secreted by activated MAIT cells, were suppressed by treatment with GSI. Moreover, both TCR-dependent MAIT cell activation by microbial-derived riboflavin intermediates and TCR-independent MAIT cell activation driven by IL-18 in synergy with IL-12, were blocked by GSI treatment.

Conclusion: Notch signaling is operating in MAIT cells and is involved in their activation both in a TCR-independent and -dependent manners.

Key words: MAIT cells, Notch signaling, gamma secretase inhibitor, MAIT cell activation, MAIT cell function

\section{From:}

${ }^{1}$ Center of Excellence in Immunology and Immune-mediated diseases, Department of Microbiology, Faculty of Medicine,

Chulalongkorn University, Bangkok, Thailand

2 Immunology Division, Department of Microbiology,

Faculty of Medicine, Chulalongkorn University, Bangkok, Thailand

Department of Microbiology, Faculty of Science, Chulalongkorn

University, Bangkok, Thailand

\section{Corresponding author:}

Pimpayao Sodsai

Department of Microbiology, Faculty of Medicine,

Chulalongkorn University, Bangkok 10330, Thailand

E-mail: pimpayao.s@chula.ac.th,yokpim@gmail.com

\section{Abbreviations:}

MAIT Mucosal-associated invariant T cells

MR1 MHC-related protein 1

MHC major histocompatibility complex

TCR T cell receptor

DLL1 Delta-like 1

DLL3 Delta-like 3

$\begin{array}{ll}\text { Abbreviations (Continued): } \\ \text { DLL4 } & \text { Delta-like } 4 \\ \text { PBMCs } & \text { peripheral blood mononuclear cells } \\ \text { FBS } & \text { fetal bovine serum } \\ \text { IL-2 } & \text { interleukin-2 } \\ \text { IL-12 } & \text { interleukin-12 } \\ \text { IL-17 } & \text { interleukin-17 } \\ \text { IL-18 } & \text { interleukin-18 } \\ \text { TNF- } \alpha & \text { tumor necrosis factor alpha } \\ \text { IFN- } \gamma & \text { Interferon gamma } \\ \text { GSI } & \text { gamma secretase inhibitor }\end{array}$

\section{Introduction}

Mucosal-associated invariant T (MAIT) cells are an innate-like $\mathrm{T}$ cell population that are distributed in blood $(\sim 5 \%$ of $\mathrm{T}$ cells) and mucosal sites, such as the respiratory tract and intestinal tract, and are also enriched in the liver $(\sim 10 \%$ of $\mathrm{T}$ cells). ${ }^{1,2}$ These cells are defined by the expression of the canonical semi-invariant TCR $\alpha$ chain containing Va7.2-Ja33, ${ }^{3,4}$ 
and are restricted to the non-classical polymorphic major histocompatibility complex (MHC) class Ib antigen-presenting molecule, MHC-related protein 1 (MR1). ${ }^{3,5,6}$ Moreover, riboflavin metabolites produced from bacteria and yeast can activate MAIT cells. ${ }^{7}$ MAIT cells can be activated in an MR1-dependent manner by riboflavin-producing microbes and in a cytokine-dependent manner by interleukin-12 (IL-12) and interleukin-18 (IL-18) that does not require MR1. ${ }^{8-10}$

MAIT cells recognize and respond to microbial infection by producing a wide range of cytokines (IFN- $\gamma$, TNF- $\alpha$, IL-17) and cytotoxic molecules (perforin, granzymes, granulysin). ${ }^{11,12}$ Moreover, cytokines produced from MAIT cells can aid in the function of effector $\mathrm{T}$ cells. MAIT cells respond to a broad range of bacteria, including Mycobacterium tuberculosis, Escherichia coli, Klebsiella pneumoniae, Pseudomonas aeruginosa, Staphylococcus aureus and yeasts. ${ }^{13,14}$ Several studies provided strong in vivo evidence of the role of MAIT cells in protection against bacterial pathogens. ${ }^{13,15,16}$ In humans, there are observations that link MAIT cell frequencies and their functions in the periphery of hepatitis C, HIV and TB patients that demonstrated the relationship and interaction between the microbiota and MAIT cells. ${ }^{17-19}$ However, the regulatory mechanisms regarding MAIT cell effector functions are not yet well studied and need more characterization.

Notch signaling is an evolutionarily conserved pathway across species that was first discovered in Drosophila melanogaster as a neurogenic gene. ${ }^{20}$ In mammals, there are four Notch receptors: Notch1, Notch2, Notch3 and Notch4 that are activated by five canonical Notch ligands: Jagged1, Jagged2, Delta-like1 (DLL1), Delta-like3 (DLL3) and Delta-like4 (DLL4). Notch receptor-ligand interaction activates sequential proteolytic cleavage by gamma-secretase on the Notch receptor itself, in order to release the Notch intracellular domain (NICD) for translocation to the nucleus. The NICD engages the nuclear binding protein CSL and upregulates transcription of specific target genes. ${ }^{21}$ Gamma secretase inhibitors (GSIs) are often used to interrogate the involvement of Notch signaling in cellular process. Notch signaling contributes to many steps of embryonic development and tissue homeostasis in organ systems as well as cell fate decisions of hematopoietic stem cells in organisms. ${ }^{22}$ Abrogation of Notch1 or Notch2 signaling leads to death at the stage of embryonic development. ${ }^{23}$ In $\mathrm{T}$ cells, Notch signaling is required for $\mathrm{T}$ lineage commitment in the thymus and $\mathrm{T}$ cell effector functions in the periphery. Notch1 or Notch3 overexpression in the thymocytes results in T-cell leukemia. ${ }^{24,25}$ Notch signaling pathway is well characterized in controlling activation, differentiation and function of peripheral blood T cells. ${ }^{26,27}$ Development of MAIT cells at least in vitro does not require Notch signaling, as opposed to conventional T cells. ${ }^{28}$ However, the role of Notch signaling pathway may play in MAIT cells has yet to be investigated. Thus, in this study, we reported the expression profiles of Notch receptors and ligands in MAIT cells and provide evidence indicating an essential role of Notch signaling in MAIT cells in response to stimuli.

\section{Materials and Methods Subject recruitment}

This study was approved by the Institutional Review Board of the Faculty of Medicine, Chulalongkorn University (COA No.515/2018). Eleven healthy donors were recruited and they gave written informed consent. All healthy donors contained normal physical examination results and no HIV infection.

\section{Peripheral blood mononuclear cell isolation}

PBMCs from whole blood were isolated. Diluted whole blood was layered on Ficoll-Hypaque reagent (Robbins Scientific Corporation, Sunnyvale, CA). PBMCs were isolated by density-gradient centrifugation at $1,500 \mathrm{rpm}$ for 30 minutes at room temperature. The separated PBMCs were collected and washed twice with RPMI1640 (Life Technologies). PBMCs were cryopreserved in 10\% DMSO in fetal bovine serum (FBS) and stored in liquid nitrogen for further experiments.

\section{Results \\ Expression profiles of Notch receptors and ligands in human MAIT cells}

To investigate whether Notch signaling is involved in regulating activation and/or effector functions of MAIT cells, we firstly characterized the expression profiles of Notch receptors/ligands in MAIT cells. The representative flow cytometry plots and the gating strategy to distinguish MAIT cells and conventional $\mathrm{T}$ cells are shown in Figure 1A. MAIT cells were identified using double positive marker of the $\mathrm{V} \alpha 7.2$ and CD161 (Figure 1A).

PBMCs from 11 healthy donors were pretreated with GSI or DMSO (vehicle control) for 1 hour before stimulating with anti-CD3/CD28 beads for 24 hours. Notch receptors (Notch $1,2,3$ and 4) were evaluated for their cell surface expression. Under resting condition, Notch 2 was predominantly detected in MAIT cells (9.4\%) at higher percentages than other Notch receptors $($ Notch $1=3.6 \%$, Notch $3=1.9 \%$, Notch $4=2.7 \%$ ), while conventional $\mathrm{CD}^{+} \mathrm{T}$ cells and $\mathrm{CD}^{+} \mathrm{T}$ cells rarely expressed any Notch receptors (Figure 1B). Interestingly, the frequency of Notch1- and Notch2-expressing MAIT cells significantly increased upon stimulation to $16.2 \%$ and $15.3 \%$, respectively. However, the frequency of Notch3- and Notch4-expressing MAIT cells did not significantly change with the stimulation (Figure 1B). As expected, the levels of Notch1 and Notch 2 were also upregulated on conventional $\mathrm{CD}^{+} \mathrm{T}$ cells and $\mathrm{CD}^{+} \mathrm{T}$ cells, as previously reported. ${ }^{29}$ Upon activation, the frequency of Notch2-expressing $\mathrm{T}$ cells was significantly higher in MAIT cells than in $\mathrm{CD}^{+} \mathrm{T}$ cells, while the frequency of Notch1-expressing cells was significantly higher in $\mathrm{CD} 8^{+} \mathrm{T}$ cells than in MAIT cells and $\mathrm{CD} 4^{+} \mathrm{T}$ cells (Figure 1C).

In the presence of GSI, the expression of Notch1 and Notch2 on MAIT cells and conventional T cells were diminished under stimulating condition (Figure 1B), suggesting that Notch signaling may regulate its own expression and that Notch signaling may have an important role in conventional $\mathrm{T}$ cells and MAIT cells during effector response. Vehicle control DMSO treatment did not affect the expression profiles of Notch receptors. 
A.
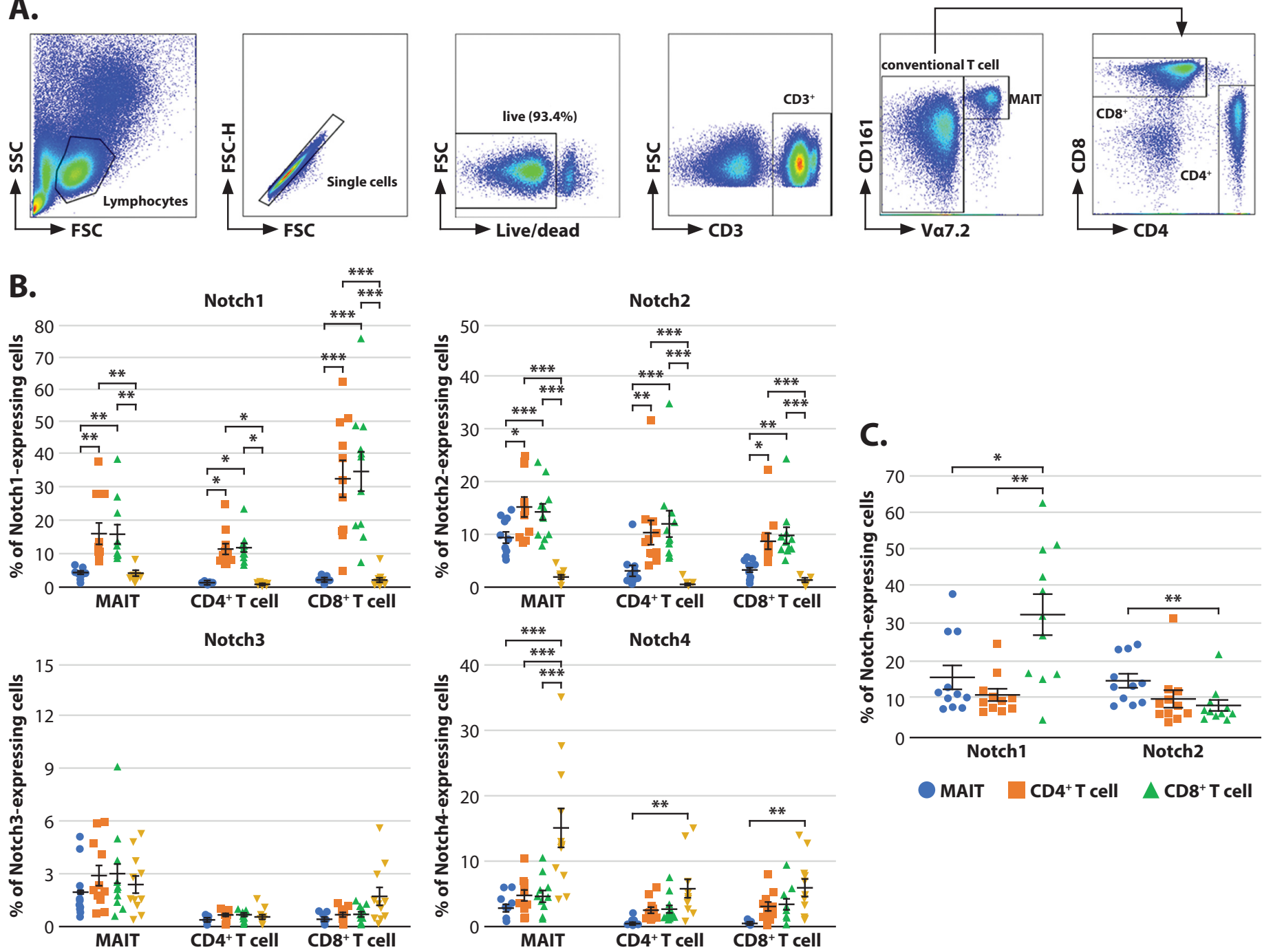

unstimulated

stimulated

stimulated + DMSO

$\nabla$ stimulated + GSI

D.
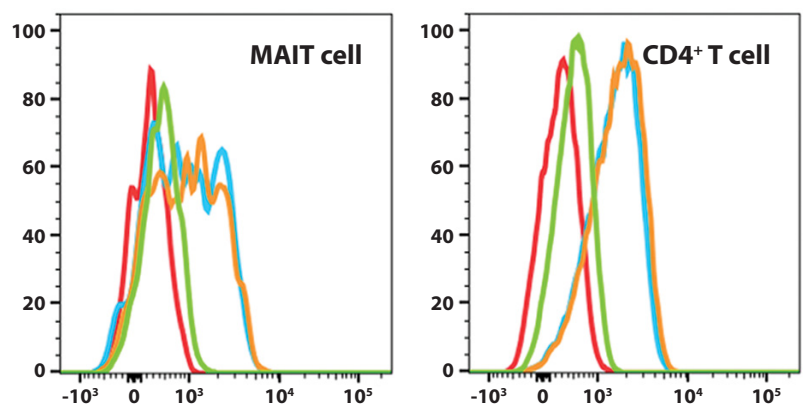

Cleaved Notch1 (mN1A)

unstimulated $\quad$ stimulated $\quad$ stimulated + DMSO

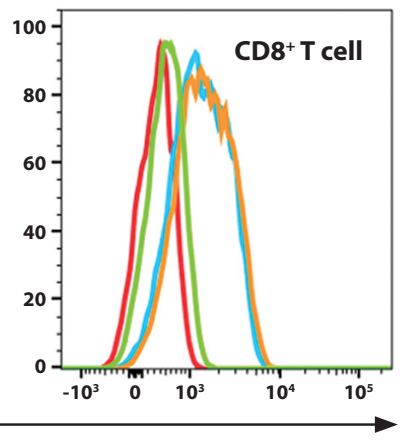

- stimulated + GSI

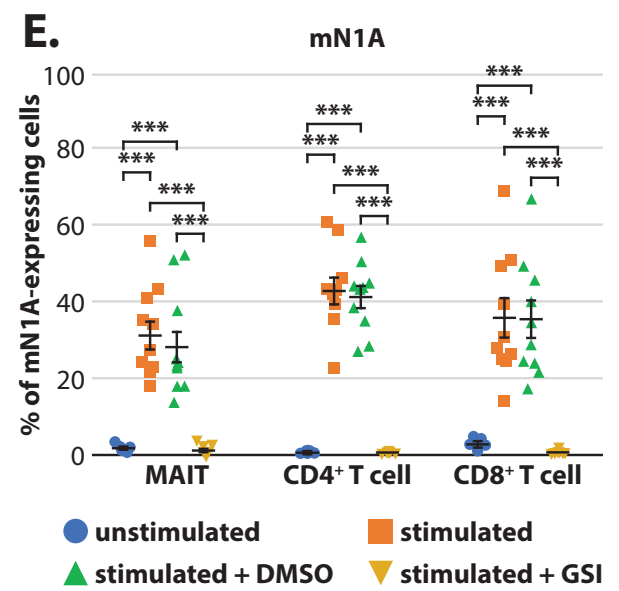

Figure 1. Activated MAIT cells are capable of expressing Notch receptors. (A) Representative dot plots and gating strategy of MAIT cells, CD4 ${ }^{+} \mathrm{T}$ cells and $\mathrm{CD}^{+} \mathrm{T}$ cells in peripheral blood mononuclear cells of healthy donors were shown. (B) Healthy PBMCs $(\mathrm{n}=11)$ were stimulated with anti-CD3/CD28-coupled beads for $24 \mathrm{~h}$ and assessed Notch receptor (Notch1, 2, 3 and 4$)$ expression on MAIT cells comparing to conventional T cells by flow cytometry. Gamma secretase inhibitor (GSI) as a notch inhibitor was added for $1 \mathrm{~h}$ prior to stimulation. (C) The frequency of Notch1- and Notch2-expressing MAIT cells and others were showed upon stimulated condition. (D) Expression of cleaved Notch1 (mN1A) in MAIT cells, CD4 ${ }^{+} \mathrm{T}_{\text {cell }}$ and $\mathrm{CD}^{+} \mathrm{T}^{+}$cells were presented in representative histograms of one donor. (E) The frequency of mN1A-producing MAIT cells and others was shown. Statistic comparisons were tested using Tukey's multiple comparisons test and Man-Whitney test. ${ }^{\star} p \leq 0.05,{ }^{* *} p \leq 0.01,{ }^{* *} p \leq$ 0.001 . 
Next, we detected the cleaved form of Notch1 in MAIT cells and conventional $\mathrm{T}$ cells by determining the level of intracellular domain of Notch1, using antibody specific for the active form of Notch1 (mN1A), upon stimulation in the presence or absence of GSI. The representative histograms of $\mathrm{mN1A}^{+}$MAIT cells and $\mathrm{CD}^{+}$and $\mathrm{CD}^{+}{ }^{+} \mathrm{T}$ cells were shown in Figure 1D and the representative dot plots of $\mathrm{mN1A}$ in MAIT cells are shown in Figure 1S. The frequency of mN1A-expressing MAIT cells was significantly increased upon stimulation, compared with unstimulated conditions and this increased mN1A was attenuated in the presence of GSI (Figure 1E).

To investigate the potential ligand(s) responsible for activation of Notch signaling in activated MAIT cells, we detected four Notch ligands (Jagged1, Jagged2, DLL1 and DLL4) in resting or stimulated MAIT cells. MAIT cells dominantly express Jagged 2 and to a lesser level, DLL4, in unstimulated conditions. In activated MAIT cells, there were no changes in the level of any ligands, except that Jagged 2 was downregulated (Figure 2). Taken together, these results indicated that Notch1 and Notch2 receptors; and Notch ligands, Jagged 2 and DLL4; are expressed in MAIT cells and the Notch signaling in MAIT cells is activated upon stimulation via anti-CD3/CD28 stimulation.
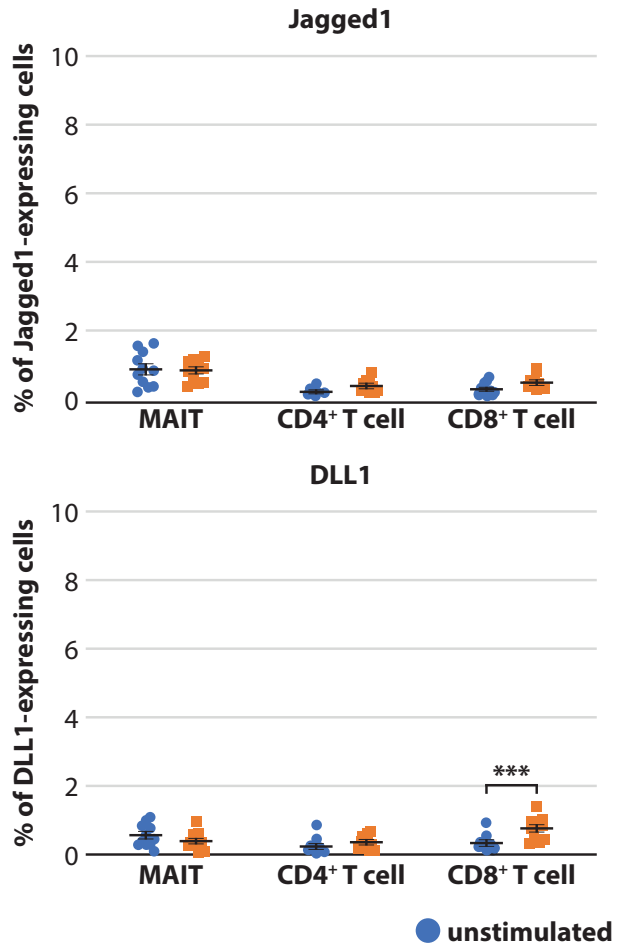

\section{Notch signaling regulates activation and function of MAIT} cells

We next tested whether the activation and function of MAIT cells are regulated through Notch signaling. PBMCs stimulated with anti-CD3/CD28 beads for 24 hours in the presence or absence of GSI were evaluated for their CD25 expression on MAIT cells. The frequency of CD25-expressing MAIT cells in the stimulated conditions were significantly higher compared to unstimulated cells (Figure 3A). The frequency of CD25-expressing MAIT cells decreased when cells were stimulated with anti-CD3/CD28 beads in the presence of GSI (Figure 3A). The effect of GSI observed in MAIT cells in this experiment was similar to that in conventional $\mathrm{T}$ cells (Figure 3A). Thus, activation of MAIT cells was compromised when Notch signaling was abrogated.

Importantly, the expression of TNF- $\alpha$, IFN- $\gamma$ and IL-17 in MAIT cells was induced with stimulation and these expressions were blocked by GSI treatment, similar to those in conventional $\mathrm{CD}^{+} \mathrm{T}$ cells and $\mathrm{CD}^{+} \mathrm{T}$ cells (Figure 3B). IL-2 expression was significantly upregulated in $\mathrm{CD}^{+}$ $\mathrm{T}$ cells, but not in MAIT cells. This finding suggested that Notch signaling may be required for TNF- $\alpha$, IFN- $\gamma$ and IL17 production in MAIT cells. Next, the role of Notch signaling in polyfunctionality of MAIT cells were analyzed.
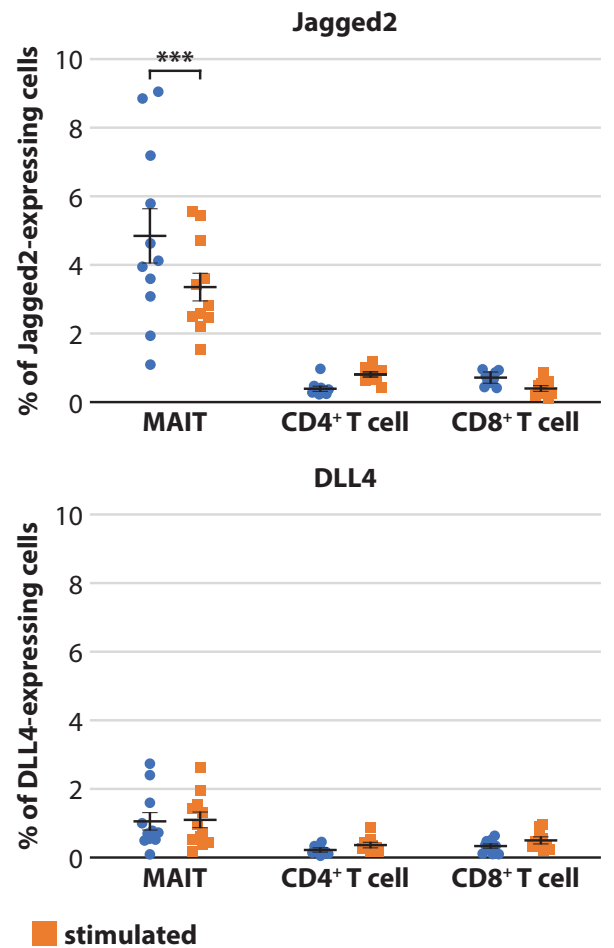

Figure 2. MAIT cells express Notch ligands. (A) Healthy PBMCs ( $\mathrm{n}=11)$ were stimulated with anti-CD3/CD28-coupled beads for 24 hours and the frequency of MAIT cells and CD4 ${ }^{+} \mathrm{T}$ cells and CD8 ${ }^{+} \mathrm{T}$ cells expressing Notch ligands (Jagged1, Jagged2, Delta-like1 and Delta-like4) was determined. Statistic comparisons were tested using Tukey's multiple comparisons test and Man-Whitney test. ${ }^{*} p \leq 0.05,{ }^{* *} p \leq 0.01,{ }^{* *} p \leq 0.001$. 

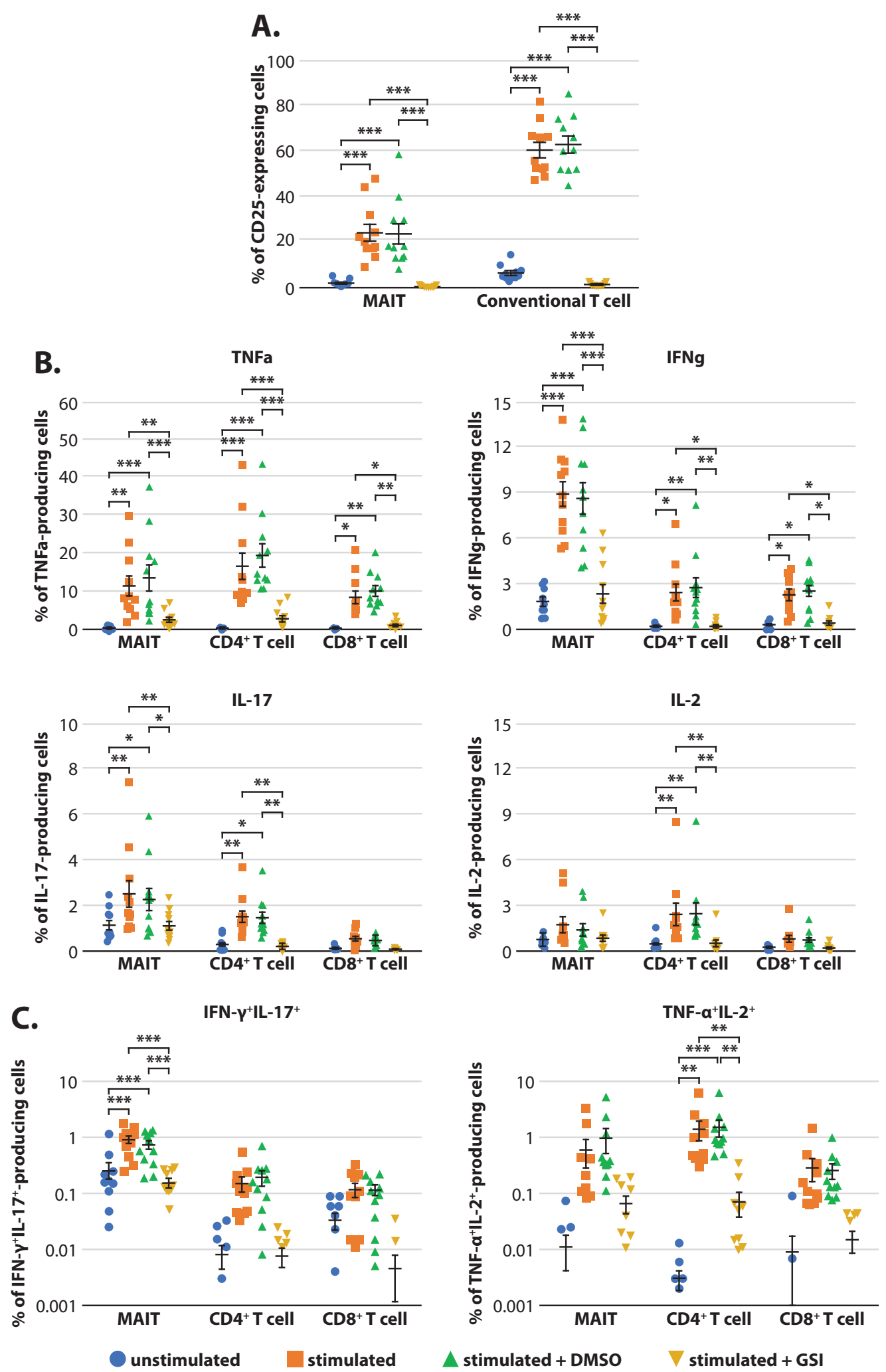

Figure 3. Regulation of MAIT cell activation and function by Notch signaling. Healthy PBMCs $(\mathrm{n}=11)$ were incubated with GSI for 1 hour before stimulating with anti-CD3/CD28-coupled beads for 24 hours and assessed activation, cytokine production and cytotoxicity of MAIT cells, CD4 ${ }^{+} \mathrm{T}$ cell and $\mathrm{CD}^{+} \mathrm{T}$ cells. (A) The frequency of activated cells was determined by CD25 expression in the presence and absence of GSI. (B) The production of cytokines; TNF- $\alpha$, IFN- $\gamma$, IL-17 and IL-2, and (D) cytotoxic molecules; granzyme B, perforin and CD107a were shown upon stimulation with the presence and absence of GSI. The polyfunctionality of (C) cytokine and (E) cytotoxic profile was assessed upon stimulation with the presence and absence of GSI. Statistic comparisons were tested using Tukey's multiple comparisons test. ${ }^{\star} p \leq 0.05,{ }^{* *} p \leq 0.01,{ }^{* *} p \leq 0.001$. 

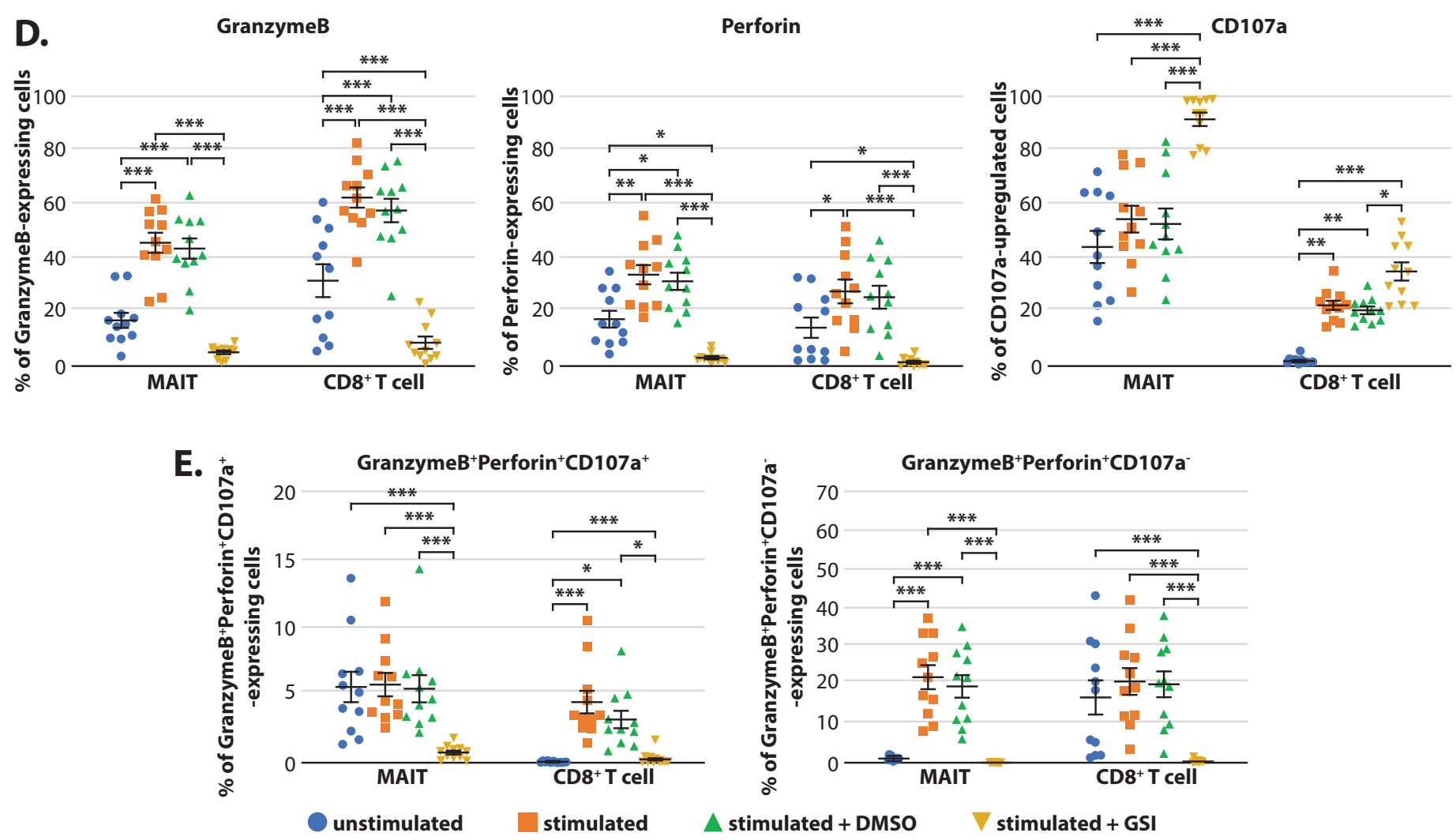

Figure 3. (Continued)

The number of IFN- $\gamma /$ IL-17 double-producing cells observed in stimulated MAIT cells significantly increased compared to unstimulated MAIT cells (Figure 3C). The frequency of TNF- $\alpha /$ IL-12 double-producing MAIT cells in stimulated conditions showed an increasing trend, but did not have significant differences compared to these in unstimulated MAIT cells, whereas the frequency of TNF- $\alpha / \mathrm{IL}-12$ double-producing $\mathrm{CD}^{+} \mathrm{T}$ cells in stimulated condition significantly increased compared to unstimulated $\mathrm{CD}^{+} \mathrm{T}$ cells. (Figure $3 C)$. The frequency of IFN- $\gamma /$ IL-17 and TNF- $\alpha /$ IL-12 double-producing MAIT cells upon stimulation were reduced in GSI-treated condition as well as these in $\mathrm{CD}^{+}$and $\mathrm{CD}^{+} \mathrm{T}$ cells (Figure 3C).

It has been reported previously that MAIT cells not only produce cytokines but also express cytotoxic molecules. In addition to Notch-mediated cytokine production in MAIT cells, we investigated the involvement of Notch signaling in the cytotoxic function of MAIT cells. The cytotoxic function of MAIT cells was measured by the expression of granzyme B, perforin and CD107a, a degranulation marker. Upon stimulation, the expressions of granzyme B and perforin in MAIT cells were significantly increased compared to unstimulated MAIT cells. Blocking Notch signaling by GSI showed a negative impact on MAIT cell cytotoxicity by reducing the expression of granzyme $\mathrm{B}$ and perforin upon stimulation (Figure 3D). On the other hand, the frequency of CD107a-expressing cells did not differ in MAIT cells with or without stimulation. This is in contrast with significantly increased CD107a in CD8 ${ }^{+} \mathrm{T}$ cells upon activation. Interestingly, CD107a significantly upregulated in the presence of GSI, both in MAIT cells and $\mathrm{CD}^{+} \mathrm{T}$ cells (Figure 3D).
To examine Notch regulation of polyfunctional cytotoxicity in MAIT cells, we found that the frequency of granzyme $\mathrm{B}^{+} /$ perforin ${ }^{+} / \mathrm{CD} 107 \mathrm{a}^{+}$expressing MAIT cells did not alter significantly upon activation while conventional $\mathrm{CD}^{+} \mathrm{T}$ cells showed an increase trend (Figure 3E). In contrast, granzyme $\mathrm{B}^{+} /$perforin ${ }^{+} / \mathrm{CD} 107 \mathrm{a}^{-}$expressing MAIT cells were significantly induced after stimulation. Moreover, polyfunctional cytotoxicity in MAIT cells was blocked by GSI treatment. Both granzyme $\mathrm{B}^{+} /$perforin ${ }^{+} / \mathrm{CD} 107 \mathrm{a}^{+}$- and granzyme $\mathrm{B}^{+} /$perforin ${ }^{+} /$ CD107a- expressing MAIT cells were significantly reduced in GSI-treated conditions (Figure 3E). Taken together, these data suggested that MAIT cell activation, cytokine production and cytotoxicity are potentially modulated by Notch signaling.

\section{Notch signaling is involved in MAIT cell activation in both TCR-dependent and TCR-independent manners}

Several studies reported that MAIT cells are activated by riboflavin-synthesizing microbes in a TCR-dependent manner and by non-riboflavin-synthesizing microbes or other inflammatory stimuli in a TCR-independent manner. ${ }^{30}$ We investigated whether those activations in MAIT cells are mediated via the Notch pathway. PBMCs were stimulated with IL-12/ IL-18 cytokines, riboflavin- or non-riboflavin-synthesizing bacterial cultured supernatant (E. coli and E. faecalis, respectively) and the frequency of CD25- and CD69-expressing MAIT cells were measured. We observed that upon activation, MAIT cells significantly increased the frequency of CD25and CD69-expressing MAIT cells by both E. coli supernatant and anti-CD3/CD28 beads compared to unstimulated conditions (Figure 4A). IL-12/IL-18 cytokines and E. faecalis supernatant could also activate MAIT cells by upregulating CD25 

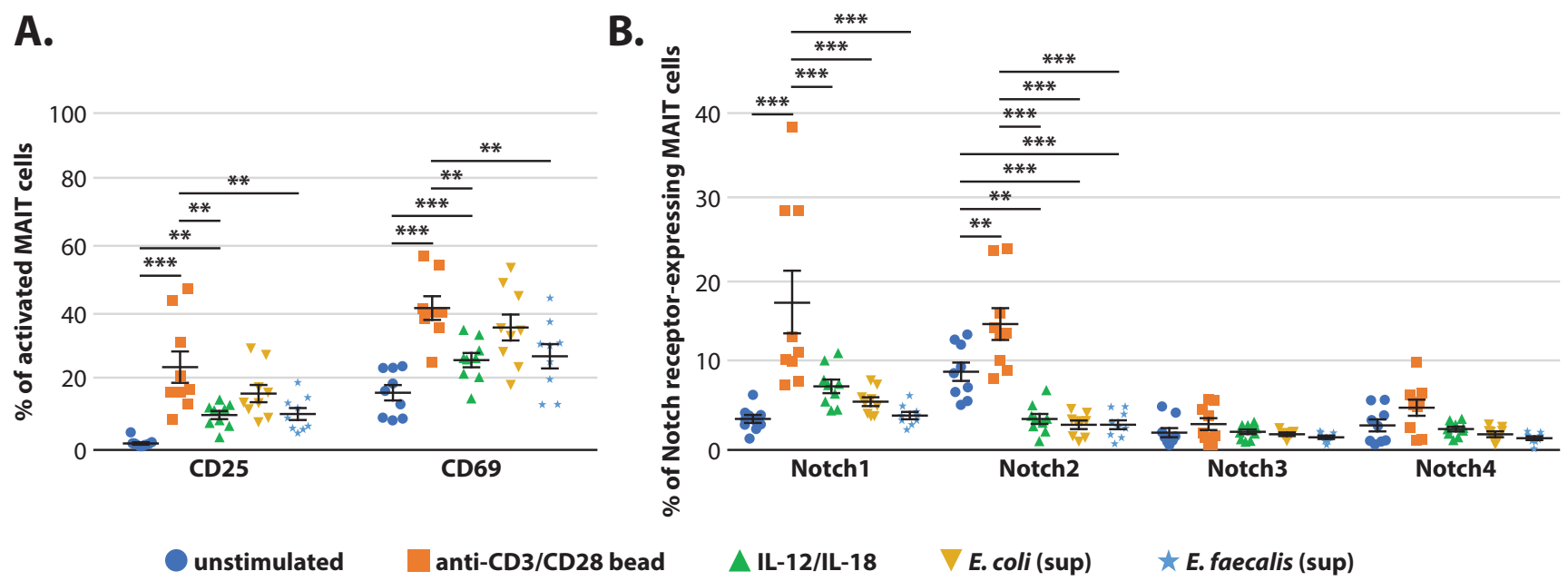

C.

mN1A

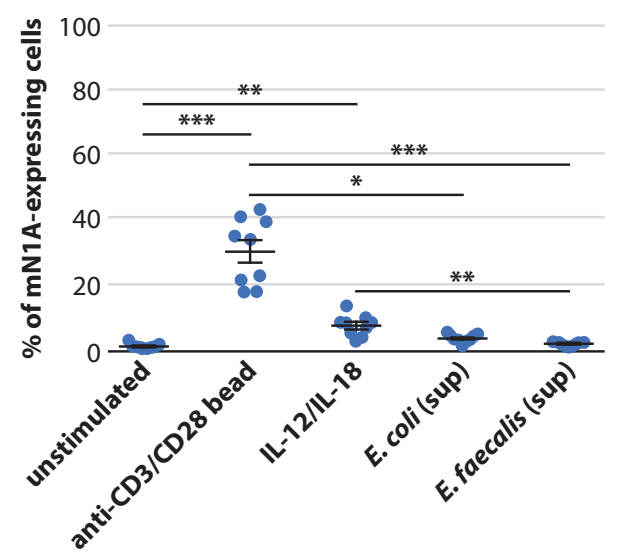

D.
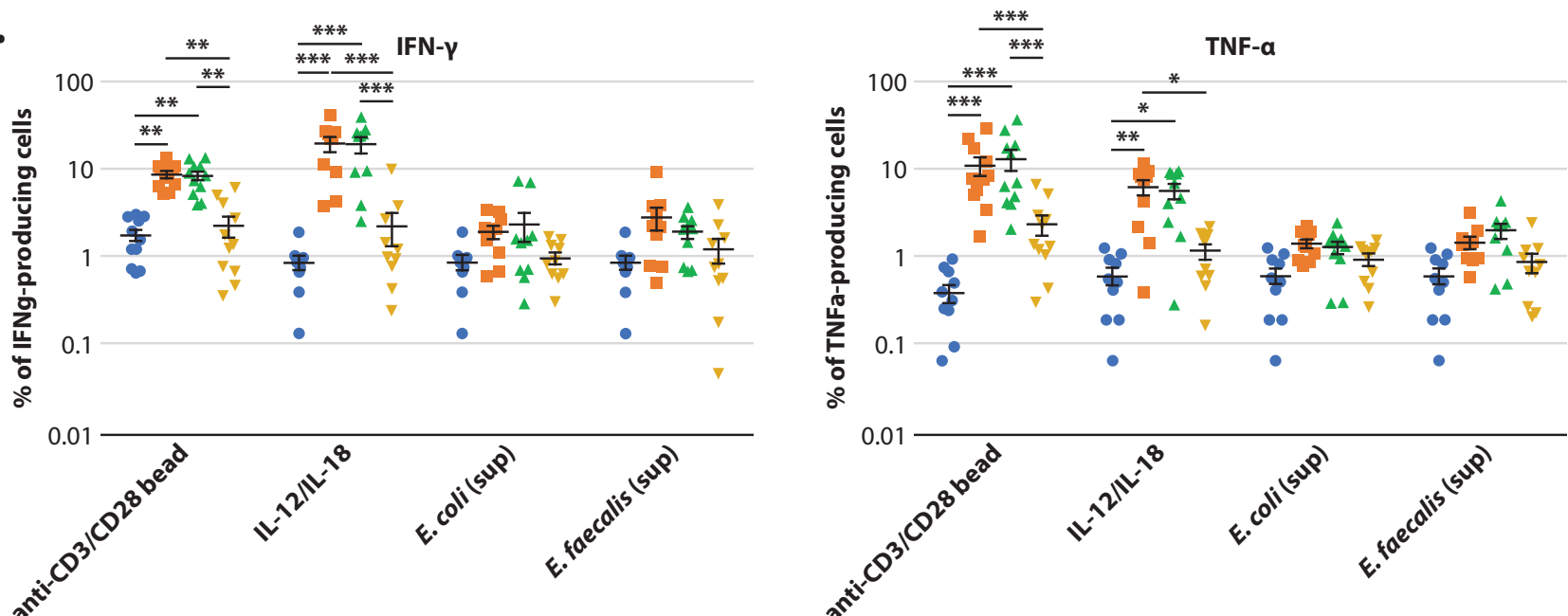

unstimulated

stimulated

$\triangle$ stimulated + DMSO

$\nabla$ stimulated + GSI

Figure 4. Notch signaling regulated both TCR-dependent and TCR-independent MAIT cell activation and cytokine production. Healthy PBMCs $(\mathrm{n}=11)$ were stimulated with anti-CD3/CD28-coupled beads, IL-12/IL-18 cytokines, E. coli supernatant or E. faecalis supernatant for 24 hours with or without GSI pretreated. (A) The frequency of activated MAIT cells was determined by CD25 and CD69 expression. (B) In the presence of stimuli, the frequency of MAIT cells expressing Notch receptor (Notch1, 2, 3 and 4) was elucidated. (C) The frequency of MAIT cells expressing mN1A was assessed. (D) IFN- $\gamma$ and TNF- $\alpha$-producing cells were shown upon stimulation in the presence and absence of GSI. (E) Pie charts depicting the proportion of TNF- $\alpha$ or IFN- $\gamma$ monoproducers and TNF- $\alpha / \mathrm{IFN}-\gamma$ double producers. Statistic comparisons were tested using Tukey's multiple comparisons test and kruskal wallis test with Dunn's multiple comparisons test. ${ }^{*} p \leq 0.05,{ }^{* *} p \leq 0.01,{ }^{* *} p \leq 0.001$. 
APJAI

E.

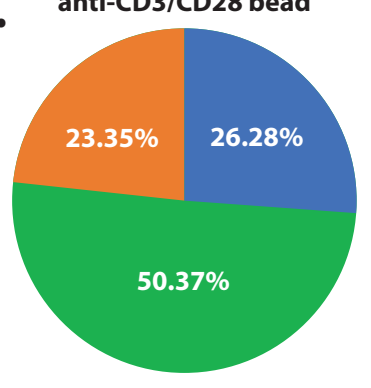

Figure 4. (Continued)
IL-12/IL-18

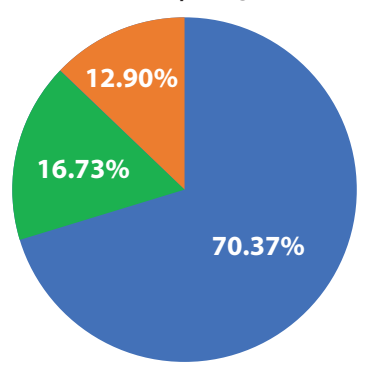

IFN- $\boldsymbol{T N F - a}$
E. coli supernatant

$5.36 \%$

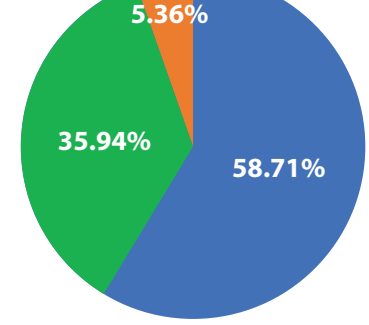

IFN- $\gamma+$ TNF-a
E. faecalis supernatant

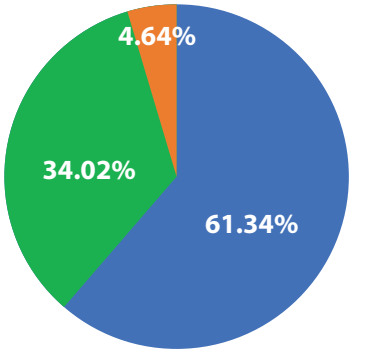

A
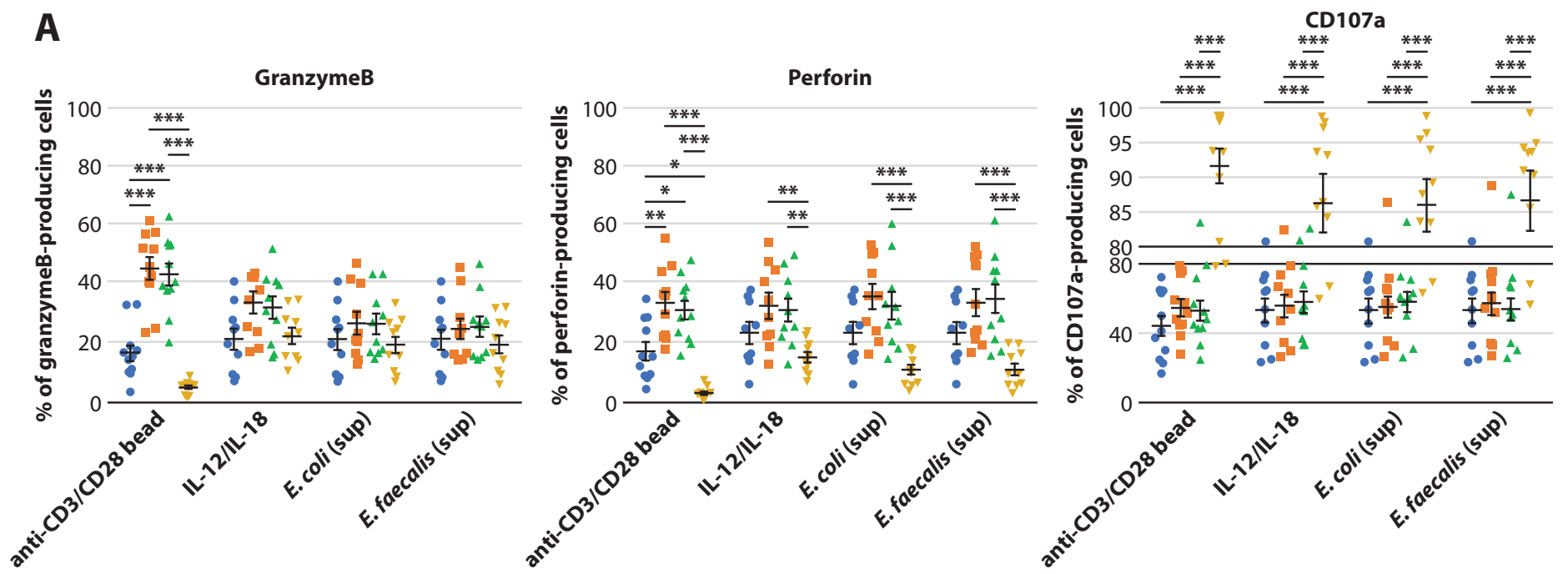

B
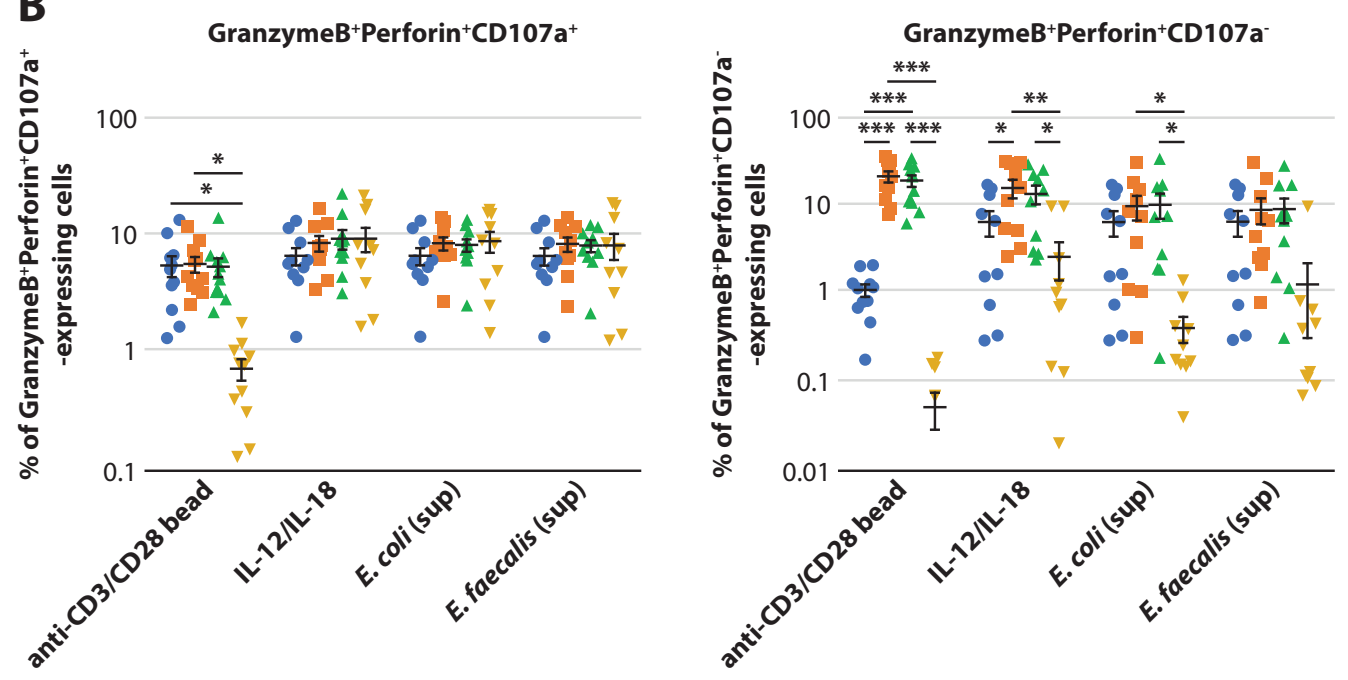

unstimulated

stimulated

$\triangle$ stimulated + DMSO

$\nabla$ stimulated + GSI

Figure 5. Notch signaling regulated cytotoxic function of MAIT cells in TCR manner and cytokine manner. Healthy PBMCs ( $\mathrm{n}=11$ ) were stimulated with anti-CD3/CD28-coupled beads, IL-12/IL-18, E. coli supernatant or E. faecalis supernatant for $24 \mathrm{~h}$ with or without GSI pretreated. (A) The cytotoxic molecules; granzyme B and perforin, and degranulation marker; CD107a, on MAIT cells were assessed. (B) The cytotoxic polyfunction of MAIT cells was illustrated. Statistic comparisons were tested using Tukey's multiple comparisons test. ${ }^{*} p \leq 0.05,{ }^{* *} p \leq 0.01,{ }^{* * *} p \leq 0.001$. 
and CD69 expression, but these upregulations were not significantly different compared to the unstimulated conditions (Figure 4A).

To determine changes in Notch receptor expressions upon TCR-dependent and TCR-independent stimuli, activated MAIT cells were analyzed. Anti-CD3/CD28 beads were able to significantly induce high Notch1 expression on MAIT cells as described above. Interestingly, the levels of Notch1 on MAIT cells stimulated with IL-12/IL-18 and E. coli supernatant were higher than the unstimulated conditions (Figure 4B). Furthermore, the frequency of $\mathrm{mN1A}$-expressing MAIT cells was significantly increased upon anti-CD3/CD28 bead and IL-12/IL-18 stimulation but not by bacterial culture supernatant (Figure 4C). Notch2 expression significantly elevated upon only anti-CD3/CD28 bead stimulation, whereas the level was significantly decreased upon IL-12/IL-18, E. coli supernatant and E. faecalis supernatant stimulation (Figure 4B). Notch 3 and Notch4 expression were not induced by any stimuli.

Next, we further analyzed whether Notch signaling mediated cytokine production upon TCR-dependent and TCR-independent stimuli or not. IFN- $\gamma$ and TNF- $\alpha$ production were observed by ICS upon stimulation with IL-12/IL-18 cytokines, E. coli supernatant and $E$. faecalis supernatant with or without GSI. The frequency of IFN- $\gamma$ - and TNF- $\alpha$-producing MAIT cells significantly increased upon stimulation by anti-CD3/ CD28 beads and IL-12/IL-18, while frequencies upon E. coli supernatant and E. faecalis supernatant activation showed increasing but not significant difference compared to unstimulated condition (Figure 4D). IL-12/IL-18 could induce IFN- $\gamma$ production $(20.1 \%)$ more than anti-CD3/CD28 beads (9\%) (Figure 4D). In addition, IFN- $\gamma$-producing cells and TNF- $\alpha$-producing cells were significantly lower in GSI-treated conditions (Figure 4D). MAIT cells stimulated with anti-CD3/CD28 beads showed the highest frequencies of TNF- $\alpha$ single expressing cells whereas MAIT cells stimulated with IL-12/IL-18, E. coli supernatant and E. faecalis supernatant showed the most proportion of IFN- $\gamma$ single expressing cells (Figure 4E).

We further analyzed whether Notch signaling mediates cytotoxic function upon TCR-dependent and TCR-independent stimulation. The expression of perforin and granzyme $B$ were significantly increased by anti-CD3/CD28 bead activation whereas those expressions had an increase trend upon activation by IL-12/IL-18, E. coli supernatant and E. faecalis supernatant, but not significantly different when compared to unstimulated conditions. Perforin production was significantly reduced in all stimuli conditions in the presence of GSI treatment. CD3/CD28 bead-induced granzyme B production was reduced in the presence of GSI treatment (Figure 5A). The level of CD107a-expressing MAIT cells did not change whether cells were activated or not (Figure 5A). Interestingly, the frequency of CD107a-expressing MAIT cells was significantly increased upon stimulation in the presence of GSI (Figure 5A). In addition, granzyme B/perforin/CD107a triple-expressing MAIT cells were not significantly upregulated upon any stimuli conditions (Figure 5B). In contrast, granzyme B/perforin double-expressing MAIT cells were significantly induced after stimulation with anti-CD3/CD28- beads and IL-12/IL-18. Moreover, polyfunctional cytotoxicity in MAIT cells activated by all stimuli conditions was blocked by GSI (Figure 5B).

In summary, the activation of MAIT cells in terms of cytokine production and cytotoxic molecule expression was dependent upon Notch signaling regardless of the stimuli.

\section{Discussion}

Previous studies reported that MAIT cells can be activated through TCR/MR1-dependent and TCR/MR1-independent (cytokine-mediated) manner. These stimuli result in proliferation and polarization for effector functions. ${ }^{7,11,13}$ The signaling pathway(s) involved in MAIT cell activation are studied in details and the signaling and the transcription factors involved in their activation and effector functions have some similarities with that of conventional T cells. ${ }^{31}$ TCR-dependent activation of MAIT cells induces production of pro-inflammatory cytokines and chemokines, dominated by IL-17A under the control of the transcription factor, retinoic acid-related orphan receptor gamma (ROR $\gamma \mathrm{t})$. This subset of MAIT cells shows Tc17-like phenotype. On the other hand, the TCR-independent activation mainly drives production of IFN- $\gamma$, perforin and granzyme B under the control of 2 transcription factors, promyelocytic leukemia zinc finger (PLZF) and T-bet, resulting in a Tc1-like phenotype. ${ }^{32,33}$ Because Notch signaling plays a key role in cell fate decision of hematopoietic stem cells and is required for T cell development, activation, proliferation, differentiation, function and apoptosis, we investigated the involvement of Notch signaling in activation and the expression of some key effector functions of MAIT cells. ${ }^{29}$ The results presented here convincingly revealed that Notch signaling plays a pivotal role in MAIT cell activation and effector functions.

Development of MAIT cells in the stage 1, 2 or 3 did not require Notch signaling through Delta-like1 ligand, in contrast to that in conventional $\mathrm{T}$ cells, but whether Notch signaling plays any role in peripheral MAIT cells is largely unknown. ${ }^{28}$ In this study, we provided evidence suggesting a critical role of Notch signaling in MAIT cell functions. Notch2 and Jagged 2 are found to be expressed higher in resting MAIT cells, compared to conventional $\mathrm{T}$ cells. Mature conventional naive $\mathrm{CD}^{+}$and $\mathrm{CD}^{+} \mathrm{T}$ cells express Notch1 and Notch2 while DLL1 and DLL4 seems to play a more dominant role. ${ }^{34}$ These results suggest that a different set of Notch receptors/ligands may be utilized in MAIT cells and conventional T cells. Because the cleaved form of Notch1 was readily detected in activated MAIT cells and GSI treatment reduced it to the basal level, we used GSI as a tool to dissect the role of Notch signaling in MAIT cells.

MAIT cell activation results in cytokine production and cytotoxic activity. ${ }^{28,35}$ The expression of activation markers (CD25 and CD69), cytokines (TNF- $\alpha$, IFN- $\gamma$, IL-17) and cytotoxic molecules (perforin, granzyme B) were all suppressed by GSI. These results strongly indicated that Notch signaling plays essential roles in regulating effector function of MAIT cells. Because the impact of GSI is profound and covered a wide range of effector functions of MAIT cells, we speculate that Notch signaling possibly controls a key step leading to MAIT cell activation. In conventional $\mathrm{T}$ cells, 
Notch signaling regulates $\mathrm{CD} 4^{+} \mathrm{T}$ cell differentiation by directly controlling the expression of master regulators such as T-bet and GATA3 ${ }^{36}$ In CD8 ${ }^{+}$T cells, Notch signaling controls the decision to develop into terminally-differentiated effector cells or memory cells and also their key cytotoxic function. ${ }^{37}$ Notch signaling controls $\mathrm{T}$ cell activation via the Akt and mTOR pathways, and via metabolic reprogramming. ${ }^{29}$ MAIT cells also utilize mTORC1/mTORC2 pathway to regulate effector lineage differentiation, especially IFN- $\gamma$-producing MAIT1 cells and IL-17-producing MAIT17 cells. ${ }^{38}$ Whether MAIT cell activation and functions are also regulated by Notch signaling in collaboration with AKT/mTOR pathways need further investigation.

We observed an interesting result where the expression of CD107a, which is a degranulation marker in MAIT cells increased in the presence of GSI. This is in contrast to the reduced cytotoxic function of MAIT cells in the presence of GSI. There was a previous report that GSI treatment did not change CD107a expression in CD8 ${ }^{+} \mathrm{T}$ cells. ${ }^{39}$ In MAIT cells, Notch signaling or other gamma-secretase dependent molecules may negatively regulate degranulation process or other GSI substrates may be responsible for this increased CD107a. Our current data revealed that Notch signaling is involved in the activation and function of MAIT cells.

There are two different pathways to trigger activation of MAIT cells, the TCR/MR1-dependent and TCR-independent mechanisms. TCR/MR1-dependent activation requires the recognition of MR1-ligands (riboflavin) by TCR. TCR-independent activation involves cytokines and engagement of TLR ligands, that can result in proliferation, secretion of related cytokines and cytotoxic function..$^{9,10,30,40}$ We found that activation of MAIT cells was dependent upon Notch signaling regardless of the stimuli. Among the stimuli tested, anti-CD3/CD28 stimulation showed strongest activation of Notch signaling. Cleaved Notch1 was upregulated upon anti-CD3/CD28 bead and IL-12/IL-18 stimulation, while Notch2 was increased in only anti-CD3/CD28 stimulated condition. IL-12/IL-18 increased cleaved Notch1 at higher level than riboflavin-dependent pathway and the use of GSI has only minimal effect on cytokine production. The impact of GSI is more profound against cytotoxic functions of MAIT cells. Whether different Notch receptor/ligand pairs are required for activation by different stimuli or not is not known. Interestingly, the expression of Notch4 on MAIT cells and conventional $\mathrm{T}$ cells was increased in the presence of GSI. The roles of Notch4 in T cells are not well documented. The signaling of Notch4 and its ligand interaction suppresses the expression of cell cycle components of lymphatic endothelial cells. ${ }^{41}$

In this study, we used GSI as a tool to study the potential role of Notch signaling in activation and function of MAIT cells. The use of GSI has some limitations as there are multiple substrates for enzymatic activity of gamma secretase, including APP, CD44 and Notch. ${ }^{42}$ To specifically narrow down whether Notch signaling is responsible for all the observed GSI effects in MAIT cells, other approaches may be needed, such as antibody blocking or gene silencing. Because conventional $\mathrm{T}$ cells and MAIT cells utilize Notch signaling in controlling their activation and functions, the use of systemic tools to control Notch signaling may need to take into consideration the impact of such manipulation on both conventional T cells and MAIT cells. If MAIT cells and conventional T cells use different sets of Notch receptors/ligands, it will open up a possibility of selective interference of MAIT/ conventional $\mathrm{T}$ cell effector functions. The functions of MAIT cells in both cytokine production and cytotoxic molecule secretion were suppressed by GSI can be intrinsic or extrinsic effect. We speculate that activation of Notch signaling in MAIT cells directly regulate the effector functions we observed because intracellular Notch1 was evident in activated MAIT cells. However, because we did not purify MAIT cells for GSI treatment, we cannot rule out the potential indirect effect of Notch signaling in other white blood cells in PBMCs that may regulate MAIT cell functions. Whether the effect we observed with GSI treatment on MAIT cells is an intrinsic effect or not needs further investigation by using purified MAIT cells.

Collectively, we report here for the first time that Notch signaling is active in activated MAIT cells and manipulating Notch signaling in MAIT cells may provide the opportunity for controlling host immune response against infections as MAIT cells play essential roles in host defence against various pathogens.

\section{Conflict of interest}

The authors declare no commercial or financial conflict of interest.

\section{Acknowledgements}

This work was supported by TRG grant (TRG6080010), Research Grant for New Scholar Ratchadaphiseksomphot Endowment Fund, Chulalongkorn University and Grants for Development of New Faculty Staff, Ratchadaphiseksomphot Endowment Fund, Chulalongkorn University. We would like to thank Dr. Tanittha Chatsuwan from Department of Microbiology, Faculty of Medicine, Chulalongkorn University for providing bacteria.

\section{References}

1. Treiner E, Lantz O. CD1d- and MR1-restricted invariant T cells: of mice and men. Curr Opin Immunol. 2006;18(5):519-26.

2. Walker LJ, Kang YH, Smith MO, Tharmalingham H, Ramamurthy N, Fleming VM, et al. Human MAIT and CD8alphaalpha cells develop from a pool of type-17 precommitted CD8 ${ }^{+}$T cells. Blood. 2012;119(2):422-33.

3. Treiner E, Duban L, Bahram S, Radosavljevic M, Wanner V, Tilloy F, et al. Selection of evolutionarily conserved mucosal-associated invariant $\mathrm{T}$ cells by MR1. Nature. 2003;422(6928):164-9.

4. Tilloy F, Treiner E, Park SH, Garcia C, Lemonnier F, de la Salle H, et al. An invariant $\mathrm{T}$ cell receptor alpha chain defines a novel TAPindependent major histocompatibility complex class Ib-restricted alpha/ beta T cell subpopulation in mammals. J Exp Med. 1999;189(12):1907-21.

5. Huang S, Gilfillan S, Cella M, Miley MJ, Lantz O, Lybarger L, et al. Evidence for MR1 antigen presentation to mucosal-associated invariant T cells. J Biol Chem. 2005;280(22):21183-93.

6. Reantragoon R, Corbett AJ, Sakala IG, Gherardin NA, Furness JB, Chen $\mathrm{Z}$, et al. Antigen-loaded MR1 tetramers define $\mathrm{T}$ cell receptor heterogeneity in mucosal-associated invariant T cells. J Exp Med. 2013; 210(11):2305-20.

7. Kjer-Nielsen L, Patel O, Corbett AJ, Le Nours J, Meehan B, Liu L, et al. MR1 presents microbial vitamin B metabolites to MAIT cells. Nature. 2012;491(7426):717-23

8. Jo J, Tan AT, Ussher JE, Sandalova E, Tang XZ, Tan-Garcia A, et al. Toll-like receptor 8 agonist and bacteria trigger potent activation of innate immune cells in human liver. PLoS Pathog. 2014;10(6):e1004210. 
9. Ussher JE, Bilton M, Attwod E, Shadwell J, Richardson R, de Lara C, et al. $\mathrm{CD} 161^{++} \mathrm{CD} 8^{+} \mathrm{T}$ cells, including the MAIT cell subset, are specifically activated by IL-12 + IL-18 in a TCR-independent manner. Eur J Immunol. 2014;44(1):195-203.

10. Ussher JE, van Wilgenburg B, Hannaway RF, Ruustal K, Phalora P, Kurioka A, et al. TLR signaling in human antigen-presenting cells regulates MR1-dependent activation of MAIT cells. Eur J Immunol. 2016;46(7):1600-14.

11. Le Bourhis L, Dusseaux M, Bohineust A, Bessoles S, Martin E, Premel V, et al. MAIT cells detect and efficiently lyse bacterially-infected epithelial cells. PLoS Pathog. 2013;9(10):e1003681.

12. Dusseaux M, Martin E, Serriari N, Peguillet I, Premel V, Louis D, et al. Human MAIT cells are xenobiotic-resistant, tissue-targeted, CD161hi IL-17-secreting T cells. Blood. 2011;117(4):1250-9.

13. Le Bourhis L, Martin E, Peguillet I, Guihot A, Froux N, Core M, et al. Antimicrobial activity of mucosal-associated invariant $\mathrm{T}$ cells (vol 11,pg 701, 2010). Nature Immunology. 2010;11(10):969-

14. Cowley SC. MAIT cells and pathogen defense. Cell Mol Life Sci. 2014; 71(24):4831-40.

15. Chua WJ, Truscott SM, Eickhoff CS, Blazevic A, Hoft DF, Hansen TH. Polyclonal Mucosa-Associated Invariant $\mathrm{T}$ Cells Have Unique Innate Functions in Bacterial Infection. Infection and Immunity. 2012; 80(9):3256-67.

16. Georgel P, Radosavljevic M, Macquin C, Bahram S. The non-conventional MHC class I MR1 molecule controls infection by Klebsiella pneumoniae in mice. Molecular Immunology. 2011;48(5):769-75.

17. Gold MC, Cerri S, Smyk-Pearson S, Cansler ME, Vogt TM, Delepine J, et al. Human mucosal associated invariant $\mathrm{T}$ cells detect bacterially infected cells. PLoS Biol. 2010;8(6):e1000407.

18. Gohil SK, Heo M, Schoenbaum EE, Celentano D, Pirofski LA. CD8 ${ }^{+} \mathrm{T}$ cells and risk for bacterial pneumonia and all-cause mortality among HIV-infected women. J Acquir Immune Defic Syndr. 2012;60(2):191-8.

19. Spaan M, Hullegie SJ, Beudeker BJ, Kreefft K, van Oord GW, Groothuismink ZM, et al. Frequencies of Circulating MAIT Cells Are Diminished in Chronic HCV, HIV and HCV/HIV Co-Infection and Do Not Recover during Therapy. PLoS One. 2016;11(7):e0159243.

20. Artavanis-Tsakonas S, Rand MD, Lake RJ. Notch signaling: cell fate control and signal integration in development. Science. 1999;284(5415): 770-6.

21. Radtke F, MacDonald HR, Tacchini-Cottier F. Regulation of innate and adaptive immunity by Notch. Nat Rev Immunol. 2013;13(6):427-37.

22. Radtke F, Fasnacht N, Macdonald HR. Notch signaling in the immune system. Immunity. 2010;32(1):14-27.

23. Huppert SS, Le A, Schroeter EH, Mumm JS, Saxena MT, Milner LA, et al. Embryonic lethality in mice homozygous for a processing-deficient allele of Notch1. Nature. 2000;405(6789):966-70.

24. Lin YW, Nichols RA, Letterio JJ, Aplan PD. Notch1 mutations are important for leukemic transformation in murine models of precursor-T leukemia/lymphoma. Blood. 2006;107(6):2540-3.

25. Bellavia D, Campese AF, Alesse E, Vacca A, Felli MP, Balestri A, et al. Constitutive activation of NF-kappaB and T-cell leukemia/lymphoma in Notch3 transgenic mice. EMBO J. 2000;19(13):3337-48.

26. Minter LM, Turley DM, Das P, Shin HM, Joshi I, Lawlor RG, et al. Inhibitors of gamma-secretase block in vivo and in vitro $\mathrm{T}$ helper type 1 polarization by preventing Notch upregulation of Tbx21. Nat Immunol. 2005;6(7):680-8.

27. Tanaka S, Tsukada J, Suzuki W, Hayashi K, Tanigaki K, Tsuji M, et al. The interleukin- 4 enhancer CNS-2 is regulated by Notch signals and controls initial expression in NKT cells and memory-type CD4 T cells. Immunity. 2006;24(6):689-701.

28. Koay HF, Gherardin NA, Enders A, Loh L, Mackay LK, Almeida CF, et al. A three-stage intrathymic development pathway for the mucosal -associated invariant T cell lineage. Nat Immunol. 2016;17(11):1300-11.

29. Amsen D, Helbig C, Backer RA. Notch in T Cell Differentiation: All Things Considered. Trends Immunol. 2015;36(12):802-14.

30. Godfrey DI, Koay HF, McCluskey J, Gherardin NA. The biology and functional importance of MAIT cells. Nat Immunol. 2019;20(9):1110-28.

31. Hinks TSC, Zhang XW. MAIT Cell Activation and Functions. Front Immunol. 2020;11:1014.

32. Hinks TSC, Marchi E, Jabeen M, Olshansky M, Kurioka A, Pediongco TJ, et al. Activation and In Vivo Evolution of the MAIT Cell Transcriptome in Mice and Humans Reveals Tissue Repair Functionality. Cell Rep. 2019;28(12):3249-62 e5.
33. Lamichhane R, Schneider M, de la Harpe SM, Harrop TWR, Hannaway RF, Dearden PK, et al. TCR- or Cytokine-Activated CD8(+) Mucosal -Associated Invariant T Cells Are Rapid Polyfunctional Effectors That Can Coordinate Immune Responses. Cell Rep. 2019;28(12):3061-76 e5.

34. Brandstadter JD, Maillard I. Notch signalling in T cell homeostasis and differentiation. Open Biol. 2019;9(11):190187.

35. Gherardin NA, Souter MN, Koay HF, Mangas KM, Seemann T, Stinear TP, et al. Human blood MAIT cell subsets defined using MR1 tetramers. Immunol Cell Biol. 2018;96(5):507-25.

\section{Supplemental Materials and Methods Stimulation of MAIT cells}

$3 \times 10^{5}$ cells of PBMCs were seeded in U-bottomed 96well plates and stimulated for 24 hours with or without anti-CD3/CD28-coupled beads, or IL-12 (25 ng/ml)/IL-18 (50 $\mathrm{ng} / \mathrm{ml})$, or $10 \mathrm{ul}$ of bacterial supernatant. Brefeldin A $(2 \mu \mathrm{g} /$ $\mathrm{ml}$ ) was added for the final 5 hours of incubation for intracellular cytokine detection. In some experiments which needed to block Notch signaling, $25 \mu \mathrm{M}$ of GSI were added in culture before stimulation for 1 hour.

\section{Antibodies and reagents}

Anti-human monoclonal antibodies (mAbs) including anti-CD3-PEcy7 (UCHT1), anti-CD4-PEcy5, anti-CD8-PerCP/ Cy5.5, anti-CD161-APCcy7, anti-Va7.2-FITC, anti-Notch1APC, anti-Notch3-APC, anti-Notch4-PE, anti-DLL1-PE, anti-DLL4-PE, anti-Jagged2-PE, anti-IFN $\gamma$-Alexa Flour 700, anti-IL17A-APC, anti-IL-2-APC, anti-TNFa-PE, anti-granzyme B-Alexa Flour 700, anti-perforin-APC, anti-CD25APC, anti-CD69-PE, mouse IgG2a Isotype-PE, mouse IgG1א

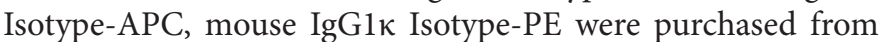
Biolegend (London, UK). Anti-human Notch2-PE and anti-human Jagged1 mAbs were obtained from BD Biosciences (Franklin Lakes, NJ, USA). Anti-human mN1A antibody was obtained from Cell Signaling Technology (Danvers, MA, USA). Live/Dead Fixable viability eFluor780 dye was purchased from eBioscience. Anti-CD3/CD28-coupled beads and brefeldin A were obtained from Invitrogen. Recombinant human IL-12 and recombinant human IL-18 were purchased from Biolegend (London, UK). Gamma-Secretase inhibitor (GSI) were purchased from Merck Millipore (USA).

\section{Bacteria}

Escherichia coli (DH5a) and Enterococcus faecalis (ATCC) were cultured in tryptic soy broth (TSB) for overnight. Bacterial supernatant was centrifuged at $4,000 \mathrm{rpm}$ for 10 minutes at $4^{\circ} \mathrm{C}$ and followed by filtration through a $0.2 \mu \mathrm{m}$ prior to use or stored at $-80^{\circ} \mathrm{C}$ for further experiment.

\section{Flow cytometry}

All experiments of flow cytometry were performed on BD LSRII (BD Biosciences). All data was analyzed using Flowjo X software (Tree Star, USA).

\section{Statistical analysis}

All data were analyzed using GraphPad Prism software version 7. Statistical significance of differences was assessed by Man-Whitney test, Tukey's multiple comparisons test and Dunn's multiple comparisons test and defined as $p \leq 0.001$ $\left.\left({ }^{* *}\right), p \leq 0.01{ }^{* *}\right)$ and $p \leq 0.05\left(^{*}\right)$. 


\section{Supplemental figure 1}
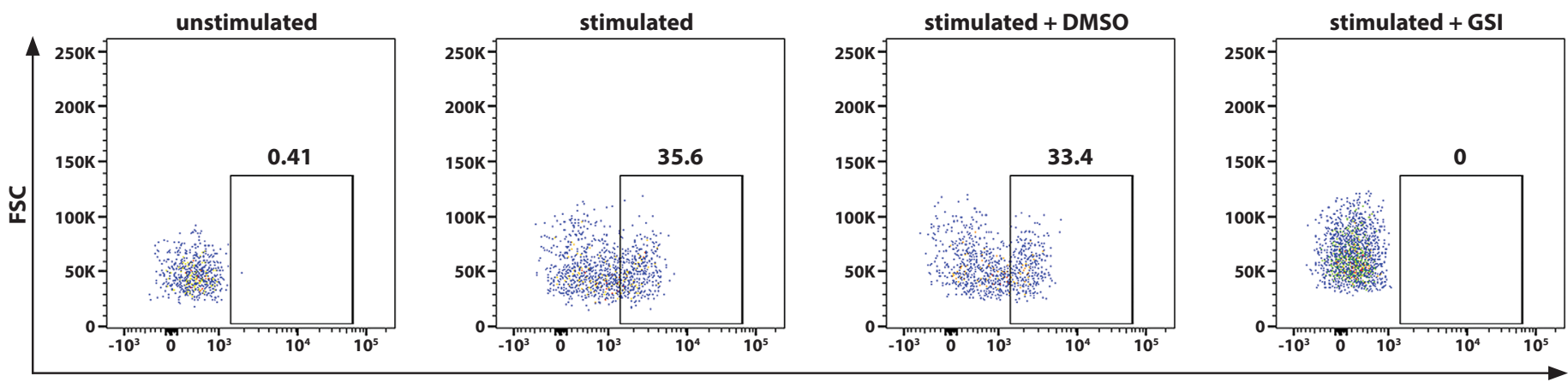

mN1A

Figure 1S. Expression of $\mathbf{m N 1 A}$ in MAIT cells. Healthy PBMCs were stimulated with anti-CD3/CD28-coupled beads for 24 hours and the expression of mN1A in MAIT cells was presented in representative dot plots of one donor.

\section{Supplemental References}

36. Osborne BA, Minter LM. Notch signalling during peripheral T-cell activation and differentiation. Nat Rev Immunol. 2007;7(1):64-75.

37. Cho OH, Shin HM, Miele L, Golde TE, Fauq A, Minter LM, et al. Notch regulates cytolytic effector function in $\mathrm{CD}^{+} \mathrm{T}$ cells. J Immunol. 2009;182(6):3380-9.

38. Tao H, Pan Y, Chu S, Li L, Xie J, Wang P, et al. Differential controls of MAIT cell effector polarization by mTORC1/mTORC2 via integrating cytokine and costimulatory signals. Nat Commun. 2021;12(1):2029.

39. Kuijk LM, Verstege MI, Rekers NV, Bruijns SC, Hooijberg E, Roep BO, et al. Notch controls generation and function of human effector $\mathrm{CD} 8^{+} \mathrm{T}$ cells. Blood. 2013;121(14):2638-46.
40. Kurioka A, Ussher JE, Cosgrove C, Clough C, Fergusson JR, Smith K, et al. MAIT cells are licensed through granzyme exchange to kill bacterially sensitized targets. Mucosal Immunol. 2015;8(2):429-40.

41. Emuss V, Lagos D, Pizzey A, Gratrix F, Henderson SR, Boshoff C. KSHV manipulates Notch signaling by DLL4 and JAG1 to alter cell cycle genes in lymphatic endothelia. PLoS Pathog. 2009;5(10):e1000616.

42. Guner G, Lichtenthaler SF. The substrate repertoire of gamma-secretase/ presenilin. Semin Cell Dev Biol. 2020;105:27-42. 